\title{
BMJ Open Socioeconomic, remoteness and sex differences in life expectancy in New South Wales, Australia, 2001-2012: a population-based study
}

\author{
Alexandre S Stephens, ${ }^{1}$ Leena Gupta, ${ }^{1}$ Sarah Thackway, ${ }^{2}$ Richard A Broome ${ }^{1}$
}

To cite: Stephens AS, Gupta L, Thackway S, et al. Socioeconomic, remoteness and sex differences in life expectancy in New South Wales, Australia, 2001-2012: a population-based study. BMJ Open 2017;7:e013227. doi:10.1136/bmjopen-2016013227

- Prepublication history and additional material is available. To view please visit the journal (http://dx.doi.org/ 10.1136/bmjopen-2016013227).

Received 28 June 2016 Revised 26 September 2016 Accepted 9 November 2016

CrossMark

${ }^{1}$ Public Health Observatory, Sydney Local Health District, Sydney, New South Wales, Australia

${ }^{2}$ Centre for Epidemiology and Evidence, NSW Ministry of Health, Sydney, New South Wales, Australia

Correspondence to Dr Alexandre S Stephens; Alexandre.Stephens@sswahs. nsw.gov.au

\section{ABSTRACT}

Objectives: Despite being one of the healthiest countries in the world, Australia displays substantial mortality differentials by socioeconomic disadvantage, remoteness and sex. In this study, we examined how these mortality differentials translated to differences in life expectancy between 2001 and 2012.

Design and setting: Population-based study using mortality and estimated residential population data from Australia's largest state, New South Wales (NSW), between 2001 and 2012. Age-group-specific death rates by socioeconomic disadvantage quintile, remoteness (major cities vs regional and remote areas), sex and year were estimated via Poisson regression, and inputted into life table calculations to estimate life expectancy.

Results: Life expectancy decreased with increasing socioeconomic disadvantage in males and females. The disparity between the most and least socioeconomically deprived quintiles was 3.77 years in males and 2.39 years in females in 2012. Differences in life expectancy by socioeconomic disadvantage were mostly stable over time. Gender gaps in life expectancy ranged from 3.50 to 4.93 years (in 2012), increased with increasing socioeconomic disadvantage and decreased by $\sim 1$ year for all quintiles between 2001 and 2012. Overall, life expectancy varied little by remoteness, but was 1.8 years higher in major cities compared to regional/remote areas in the most socioeconomically deprived regions in 2012.

Conclusions: Socioeconomic disadvantage and sex were strongly associated with life expectancy. The disparity in life expectancy across the socioeconomic spectrum was larger in males and was stable over time. In contrast, gender gaps reduced for all quintiles between 2001 and 2012, and a remoteness effect was evident in 2012, but only for those living in the most deprived areas.

\section{INTRODUCTION}

Life expectancy at birth is an important summary measure of health and well-being and is dependent on many factors. These

\section{Strengths and limitations of this study}

- Large, population-based study of life expectancy over a 12-year period (2001-2012).

- Used modelling to generate smoothed estimates of trending life expectancy.

- Underpinned by the availability of information on key sociodemographic variables which was used to estimate differences in life expectancy associated with poorer health mediated by sociodemographic inequalities.

- Ecological area-based measures of socioeconomic status likely underestimated socioeconomic gradients in life expectancy.

include biological determinants (eg, genetics, phenotype and physiology), environmental conditions (work environments, housing conditions and exposure to pollution), socioeconomic factors (income, education and employment) and risk factors related to individual behaviour (nutrition, exercise, smoking and alcohol consumption). ${ }^{1} \quad{ }^{2}$ Importantly, socioeconomic factors, such as education, income and wealth, social support, occupation, and housing, which are associated with health and mortality, ${ }^{3}{ }^{4}$ are, to varying degrees, likely to be modifiable and could be the subject of interventions designed to ameliorate their impacts or mitigate their consequences.

Australia is one of the healthiest countries in the world ranking among the top 10 of all countries for life expectancy at birth and among the top 20 for healthy life expectancy. ${ }^{5}$ Australia has a moderate level of income inequality, with a Gini coefficient of 0.326 in 2012, close to the average for Organisation for Economic Co-operation and Development (OECD) nations (0.320), and less unequal than the USA (0.401) and Great Britain (0.351), but more unequal than many European nations. ${ }^{6}$ Australia also 
benefits from a publically funded universal healthcare system (Medicare), and a subsidised, defer-payment (loan scheme) structured higher education system, facilitating improved and equitable access to quality healthcare and education.

However, despite only moderate income inequality, Australia displays substantial mortality differences across the socioeconomic spectrum. ${ }^{7}$ An Australian Institute of Health and Welfare (AIHW) report found that, between 2009 and 2011, standardised mortality rates were 20\% and $30 \%$ higher for females and males, respectively, living in the most socioeconomically disadvantaged areas compared to those living in the least disadvantaged areas. ${ }^{7}$ The same report also showed that the standardised mortality rate of males was $50 \%$ higher than that of females, and that increasing distance from major cities was associated with increasing rates of death (20-40\% higher in those living in remote and very remote areas). ${ }^{7}$

The AIHW report primarily described mortality differentials using standardised mortality rates. An alternative perspective of mortality is life expectancy, which is generally considered to be more intuitive and less challenging to interpret than mortality rates and, as it does not rely on the use of standard populations which are often unique to geographical regions, is useful for international comparisons. ${ }^{8}$ Additionally, an assessment of the extent to which socioeconomic variation was confounded by remoteness was not included in the AIHW report, and crucially, nor was an assessment of how life expectancy inequalities may have changed over time. Hence, it remains unclear how mortality differentials by key sociodemographic factors translate to differences in life expectancy, and whether life expectancy inequalities have improved, remained unchanged or perhaps worsened over time. In this study, we used deaths and population data from Australia's largest state, New South Wales (NSW), and evaluated differences in life expectancy by sex, socioeconomic status (SES) and remoteness. We also explored the patterns of change in life expectancy between 2001 and 2012 to assess whether life expectancy inequalities have changed over time.

\section{METHODS}

\section{Data sources}

NSW is Australia's largest state, accounting for approximately one-third of the total Australian population and constituting a large representative sample. The study included all NSW residents who died between 1 January 2001 and 31 December 2012. Mortality data were obtained from the Australian Coordinating Registry (ACR) Cause of Death Unit Record File (CODURF) (2007-2012), the Australian Bureau of Statistics (ABS) CODURF (2001-2006) and the NSW Register of Births, Deaths and Marriages (RBDM) (2011-2012). ACR and ABS CODURFs contain information on all deaths registered in Australia, with causes of deaths coded according to the International Statistical Classification of Diseases and Related Health Problems, 10th revision (ICD-10). ${ }^{9}$ ACR and ABS sources of deaths data were sequential and did not overlap. The NSW RBDM is a register of vital statistics of NSW residents, which includes information on deaths registered in NSW.

Year of death, age (years), sex, state of usual residence and Statistical Area Level 2 (SA2) of residence were available for ABS and ACR CODURF data. SA2s are the third smallest unit of geography in the Australian Statistical Geography Standard (ASGS). SA2s generally have populations ranging from 3000 to 25000 persons (median of 13000 in NSW), and represent communities that are socially and economically interrelated. ${ }^{10}$ SA2 of residence was used to assign an area-level measure of SES to each death record using the ABS socioeconomic indexes for areas (SEIFA) index of relative socioeconomic disadvantage (IRSD). The IRSD is a composite measure of disadvantage and consists of variables pertaining to housing, income, education, employment and occupation. ${ }^{11}$ Quintiles of the IRSD were used in the study. SA2 of residence was also used to assign an urban (major city) or regional/remote (inner and outer regional, and remote and very remote) area of residence to each death record based on the Accessibility and Remoteness Index of Australia Plus. ${ }^{12}$

NSW RBDM deaths data for the Sydney metropolitan area was obtained for 2011 and 2012. These data contained Statistical Area Level 1 (SA1) of residence. SA1 is one level smaller than SA2 (generally containing 200 to 800 persons) ${ }^{10}$ and was used to assign finer area-level measures of IRSD to death records. The NSW RBDM deaths data were used in a validation analysis to evaluate potential misclassification error-mediated bias associated with assigning IRSD at the larger SA2 level. SA2 and SA1 midyear (30 June) estimated residential populations by sex and age were obtained from the NSW Ministry of Health.

\section{Statistical methods}

Descriptive statistics on the total number of deaths and the leading causes of death by sex and year were calculated. Leading causes of death were classified according to the major categories listed in the AIHW leading causes of deaths data file, ${ }^{13}$ and were identified using ICD-10 codes for coronary heart disease (CHD) (I20-I25), cerebrovascular disease (I60-I69), dementia and Alzheimer's disease (F01, F03, G30), chronic obstructive pulmonary disease (COPD) (J40-J44), diabetes (E10-E14), cancers (C00-C49) and external causes (V00-Y99). Age-standardised mortality rates for NSW and sex-SES-remoteness strata were calculated using the Australian residential population as on 30 June 2011 as the standard population, and with age groups of $<1$ and 1-4 years, followed by 5-year intervals up to an openended interval of 85 years or more.

Traditional life expectancy at birth was estimated using a modified Chiang II method, ${ }^{14}{ }^{15}$ which additionally included a variance estimate for the final age interval as 
described previously. ${ }^{16}$ Abridged life tables were used implementing the same age groups applied in the calculation of age-standardised mortality rates. For life table calculations, we assumed that each person who died survived the following proportion of the age interval in which they died: 0.09 for age $<1$ year, 0.4 for $1-4$ years and 0.5 for all 5 -year age groups. ${ }^{15}$

A Poisson regression model-based approach was also used to estimate life expectancy. For these models, age-group-specific death rates were estimated by sex, SES, remoteness and year. A standard log-link function was applied, and death rates were modelled using fixed terms for the categorical variables age group, sex, SES and remoteness, with two-way interactions for age group and sex, age group and SES, and a three-way interaction for age group, sex and SES. The model also included year as a continuous variable, with two-way interaction terms for year and each sex, SES and remoteness, and a three-way interaction term for year, SES and remoteness. The effect of year was assumed to be linear and model parameters were estimated in OpenBUGS with independent flat or diffuse priors for model parameters. Modelled deaths rates were inputted into life table calculations as per the Chiang II method to estimate modelled life expectancy at birth via Gibbs sampling in OpenBUGS.

Agreement analysis between Chiang II and Poisson regression life expectancy estimates was carried out according to the method described by Bland and Altman. ${ }^{17}$ Owing to their very small populations, standardised mortality rates and life expectancies for IRSD quintiles 4 and 5 in regional/remote areas are not reported, although data from these areas were included in the modelling of life expectancy. Total and leading causes of deaths, age standardised mortality rates and traditional Chiang II estimates of life expectancy at birth were calculated in SAS Enterprise Guide, V.6.1 (SAS Institute, Cary, North Carolina, USA). Posterior mean estimates for Poisson regression model parameters and estimates of differences in modelled life expectancy between sex-SES-remoteness strata by year and within strata over time were calculated in OpenBUGS V.3.2.2.

\section{RESULTS}

\section{Total deaths and leading causes}

Between 2001 and 2012, the annual number of deaths increased from 21421 to 24129 (12.6\% increase) with a corresponding population rise from 3288514 to 3679612 ( $12 \%$ increase) for females, and from 23121 to 25016 deaths $(8.2 \%$ increase) with a population increase from 3241835 to 3627571 ( $12 \%$ increase) for males (table 1). In contrast, age-standardised mortality rates declined substantially, with decreases of $15.2 \%$ and $20.8 \%$ observed for females and males, respectively (table 1). Traditional life expectancy increased by 1.9 years for females and 3.0 years for males, between 2001 and 2012 (table 1).

The leading causes of deaths during the study period were CHD, cancer and cerebrovascular disease (figure 1), collectively accounting for more than 35\% and $40 \%$ of all deaths per year for females and males, respectively. The percentage of CHD deaths consistently declined over the study period in females and males. In contrast, the percentage of cancer deaths remained mostly stable, whereas the percentage of deaths caused by dementia/Alzheimer's disease more than doubled for each females and males, becoming a top 4 cause of death in females, and accounting for a comparable proportion of deaths to COPD in males (figure 1).

\section{Life expectancy differences by sex, remoteness and SES}

Traditional and model-based life expectancy estimates were calculated for different sex-SES-remoteness strata by year (see online supplementary table; figure 2). Traditional life expectancy estimates displayed local variation from year to year in addition to an underlying trend of increase over time. Modelling of life expectancy provided a mechanism to smooth this local variation while generating estimates that closely matched

Table 1 Populations, total deaths, standardised mortality rates (Rate; per 100000 ) and life expectancies at birth (Lex) by year for males and females, NSW, 2001-2012

\begin{tabular}{|c|c|c|c|c|c|c|c|c|}
\hline \multirow[b]{2}{*}{ Year } & \multicolumn{4}{|l|}{ Females } & \multicolumn{4}{|l|}{ Males } \\
\hline & Population & Deaths & Rate & Lex & Population & Deaths & Rate & Lex \\
\hline 2001 & 3288514 & 21421 & 533.1 & 83.6 & 3241835 & 23121 & 826.1 & 78.2 \\
\hline 2002 & 3315023 & 22280 & 540.3 & 83.4 & 3265784 & 23826 & 832.9 & 78.2 \\
\hline 2003 & 3337006 & 22587 & 536.1 & 83.5 & 3283709 & 23447 & 800.8 & 78.7 \\
\hline 2004 & 3353338 & 22468 & 524.7 & 83.7 & 3297397 & 23550 & 790.0 & 78.9 \\
\hline 2005 & 3376712 & 21967 & 499.9 & 84.3 & 3316494 & 23053 & 751.2 & 79.4 \\
\hline 2006 & 3403655 & 22564 & 501.1 & 84.3 & 3339035 & 23459 & 747.4 & 79.5 \\
\hline 2007 & 3447018 & 23126 & 497.4 & 84.3 & 3387138 & 24083 & 738.8 & 79.8 \\
\hline 2008 & 3498364 & 23772 & 495.5 & 84.4 & 3445097 & 24464 & 730.1 & 79.9 \\
\hline 2009 & 3550819 & 23174 & 472.0 & 85.0 & 3502936 & 24252 & 699.7 & 80.3 \\
\hline 2010 & 3596185 & 23538 & 465.5 & 85.1 & 3548107 & 24235 & 674.5 & 80.6 \\
\hline 2011 & 3633420 & 24582 & 472.3 & 84.9 & 3585109 & 25480 & 686.5 & 80.6 \\
\hline 2012 & 3679612 & 24129 & 452.2 & 85.5 & 3627571 & 25016 & 654.2 & 81.2 \\
\hline
\end{tabular}




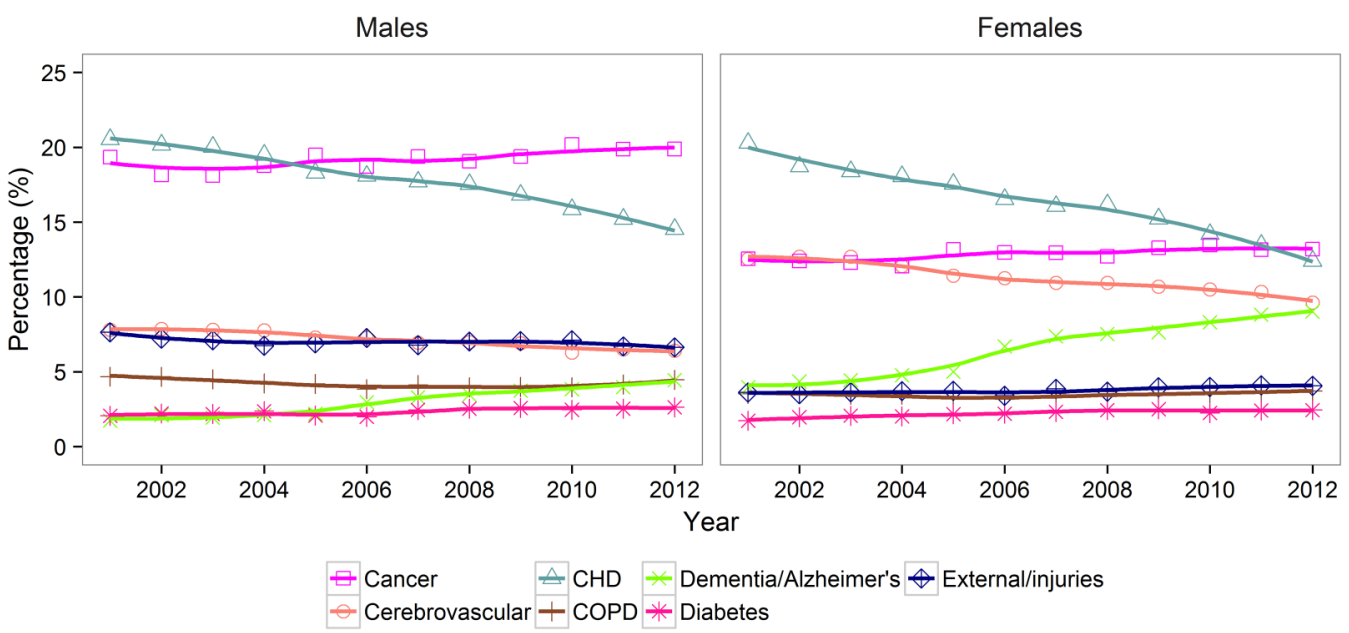

Figure 1 Leading causes of deaths by year for males and females, NSW, 2001-2012. Markers represent the annual percentage of deaths attributable to causes, and smoothed lines represent LOESS fit curves of trends over time.

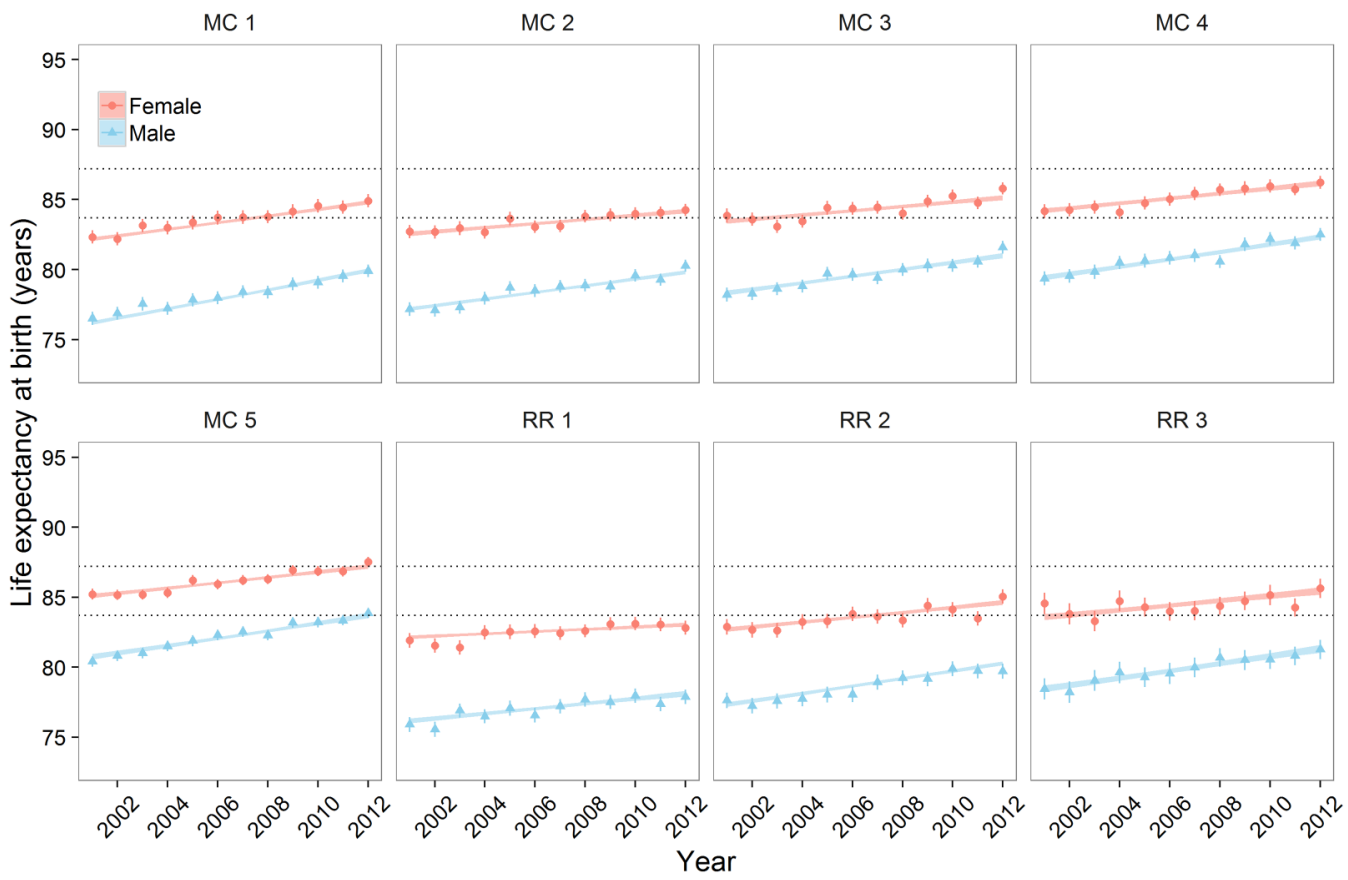

Figure 2 Trending patterns of life expectancy by sex, SES quintile and remoteness, NSW, 2001-2012. Markers (circles and triangles) represent traditional life expectancy estimates, and fit lines represent modelled (smoothed) estimates. Individual plots represent strata groups defined by remoteness category (MC as major city; RR as regional/remote) and SES quintiles (1-5).

traditional estimates, as shown by the high agreement between the two methods (see online supplementary figure S1). Model-based estimates were used to explore variation in life expectancy by sex, SES and remoteness in 2012, and, for comparative purposes allowing evaluation of how differences may have changed overtime, in 2001 (beginning of the study).

Online supplementary figure S2 shows all possible pairwise differences in life expectancy between SESremoteness strata by sex, and sex differences in life expectancy for each SES-remoteness strata, in 2001 and 2012. Differences in life expectancy by SES (differences between SES quintile 5 and all other quintiles, major city areas), sex (female estimates minus male estimates) and remoteness (major city area estimates minus rural/ remote area estimates) in 2001 and 2012 are displayed in figure 3. In 2001, clear gradients in life expectancy differences were observed with increasing IRSD quintile for males and females (figure $3 \mathrm{~A}$ and see online supplementary figure 2), although the gradient was steeper in males. For example, the estimated difference in life expectancy between quintile 5 and quintile 1 in major cities was 4.54 (95\% CI (4.37 to 4.74$)$ ) years for males and 2.91 (2.74 to 3.10 ) years for females. In 2012, the gradients in life expectancy differences with increasing IRSD quintile remained, displaying similar patterns 

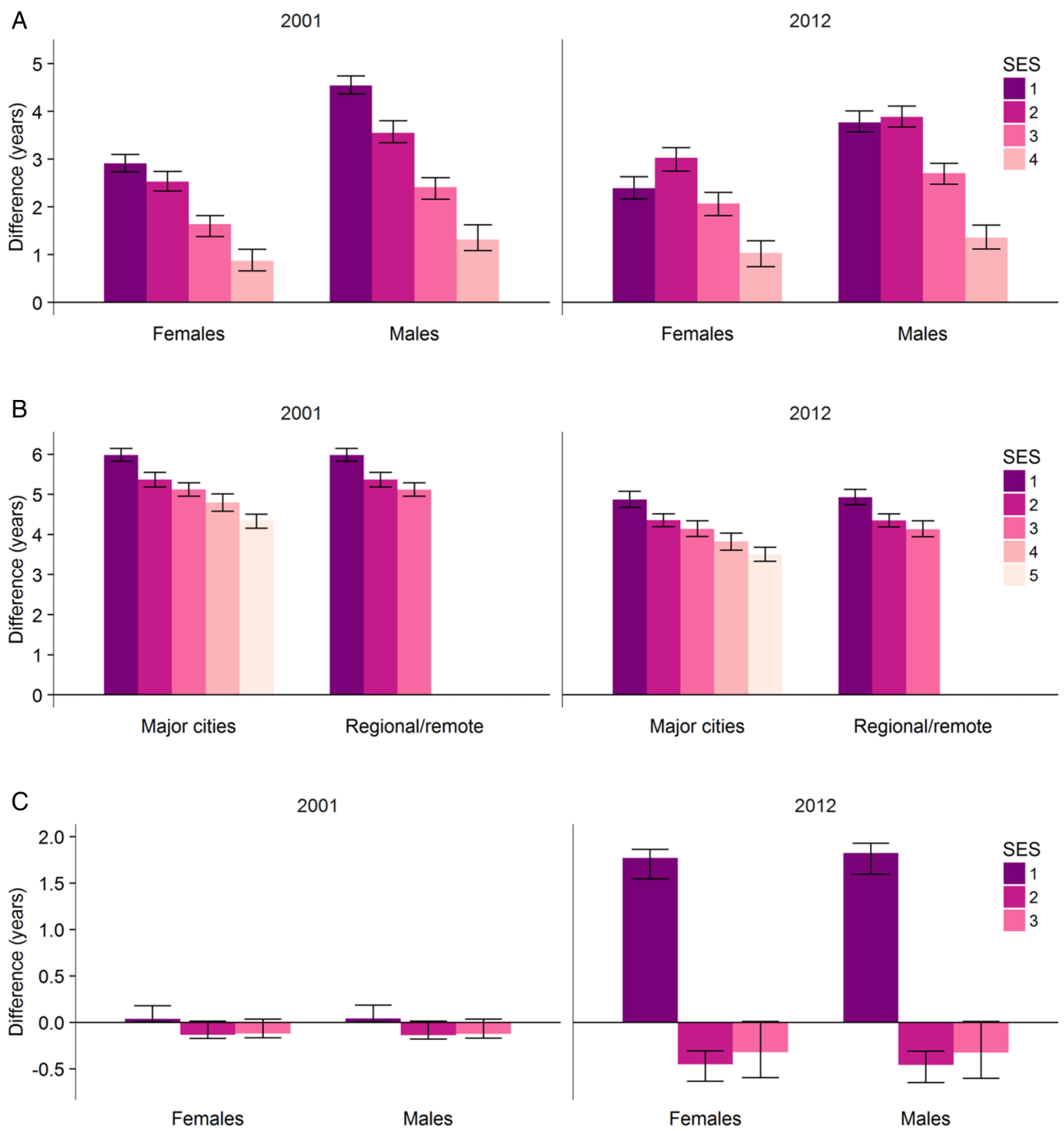

Figure 3 Differences in life expectancy by SES, sex and remoteness, NSW, 2001 and 2012. (A) Differences in modelled life expectancy between SES quintile 5 (top quintile) and SES quintiles 1-4, for males and females in major city areas, 2001 and 2012. (B) Sex differences in modelled life expectancy (female estimates minus male estimates) by SES-remoteness strata, 2001 and 2012. (C) Differences in modelled life expectancy between major city areas and rural/remote areas by SES quintile (data permitting), 2001 and 2012. Error bars represent 95\% credible intervals.

to that observed in 2001. Exceptions to the patterns were seen for major city, IRSD quintile 1, which globally showed net gains of $\sim 1$ year relative to all other SES-remoteness strata (thus reducing the size of the difference relative to quintile 5), and regional/remote, IRSD quintile 1, which displayed net decreases of $\sim 1$ year compared to all other SES-remoteness strata over the study period (figure $3 \mathrm{~A}$ and see online supplementary figure S2).

Gender gaps in life expectancy ranged from 4.35 to 5.98 years in 2001, and were conditional on IRSD quintile such that the magnitude of the differences decreased with increasing SES (figure 3B). In 2012, sex differences in life expectancy persisted, although the scale of the differences decreased by $\sim 1$ year across all strata groups (sex differences now ranged from 3.50 to 4.93 years). The size of the differences in life expectancy by sex remained related to IRSD quintile. In 2001, remoteness had little effect on life expectancy, with estimates of differences ranging from 0.4 to -0.14 years for males and females between remoteness groupings defined by equivalent IRSD quintiles (eg, estimated difference of 0.04 years $(0.01-0.18)$ for quintile 1 females; figure 3C). However, in 2012, a clear remoteness effect 


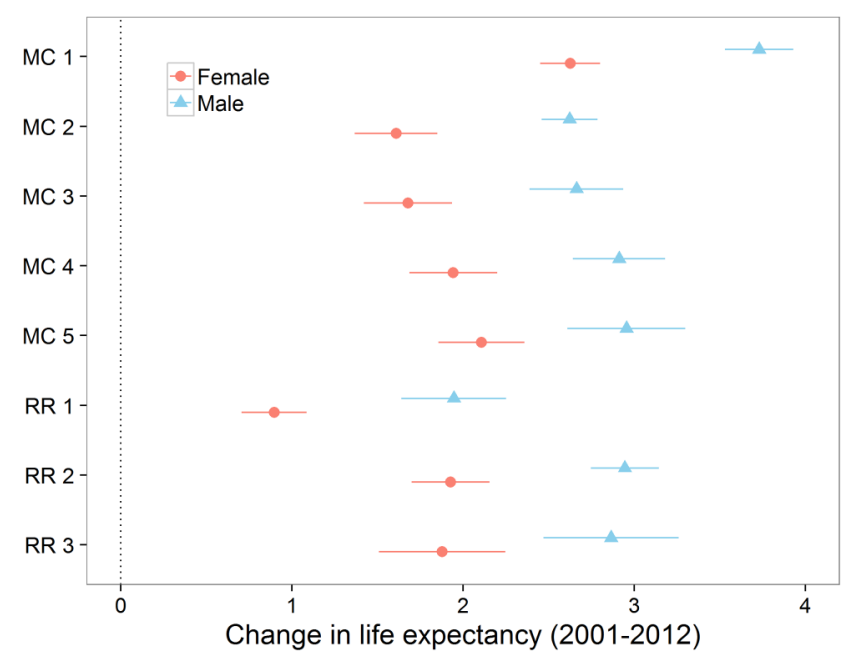

Figure 4 Gains in life expectancy between 2001 and 2012 for SES-sex-remoteness strata. Error bars represent 95\% credible intervals. MC, major city; RR, regional/remote areas; $1-5$, SES quintile.

was now evident for IRSD quintile 1, where life expectancy was 1.77 (1.54 to 1.86 ) and 1.82 (1.60 to 1.93 ) years higher for females and males, respectively, in major cities compared to regional/remote areas. In contrast, life expectancy in IRSD quintiles 2 and 3 were slightly higher in regional/remote areas relative to equivalent IRSD quintiles in major cities, for males and females.

Assessment of gains in life expectancy between 2001 and 2012 revealed that IRSD quintiles 2, 3, 4 and 5 experienced similar, sex-specific increases, ranging from 1.6 to 2.1 years for females, and 2.6 to 3.0 years for males (figure 4). On the other hand, gains were higher for IRSD quintile 1 in major cities (2.63 (2.42 to 2.79) for females, and 3.73 (3.60 to 3.89) for males), but lower for IRSD quintile 1 in regional/remote areas $(0.90$ (0.71 to 1.10$)$ for females, and 1.95 (1.78 to 2.19) for males (figure 4). Across all strata groups, the gains in life expectancy for males were higher than females (figure 4).

\section{Validation substudy}

All results above were calculated using an ecological measure of IRSD assigned at SA2-level. The degree of bias caused by misclassification of IRSD related to the use of SA2-level IRSD information was estimated by recalculating life expectancy at birth for the Sydney metropolitan area using SA1-level IRSD quintiles and comparing to estimates obtained using SA2-level IRSD. The analysis showed that gradients in life expectancy across quintiles were steeper for SA1-based estimates, particularly for males (figure 5A). Assessment of the differences between SA1-based and SA2-based estimates by quintile showed that life expectancy was overestimated in quintile 1 for males and females by 1.4 and 0.8 years, respectively, in 2012 (figure 5B), when using SA2-level IRSD data. In contrast, life expectancy was underestimated in by about 0.7 years in quintile 5 for males, and by about 0.5 years in quintile 2 for females (figure $5 \mathrm{~B}$ ) in 2012 with SA2-level information. Similar differences were observed in 2001.

\section{DISCUSSION}

In this study, we assessed the effects of sex, socioeconomic disadvantage and remoteness on life expectancy at birth and evaluated the extent to which these key sociodemographic factors affected gains in life
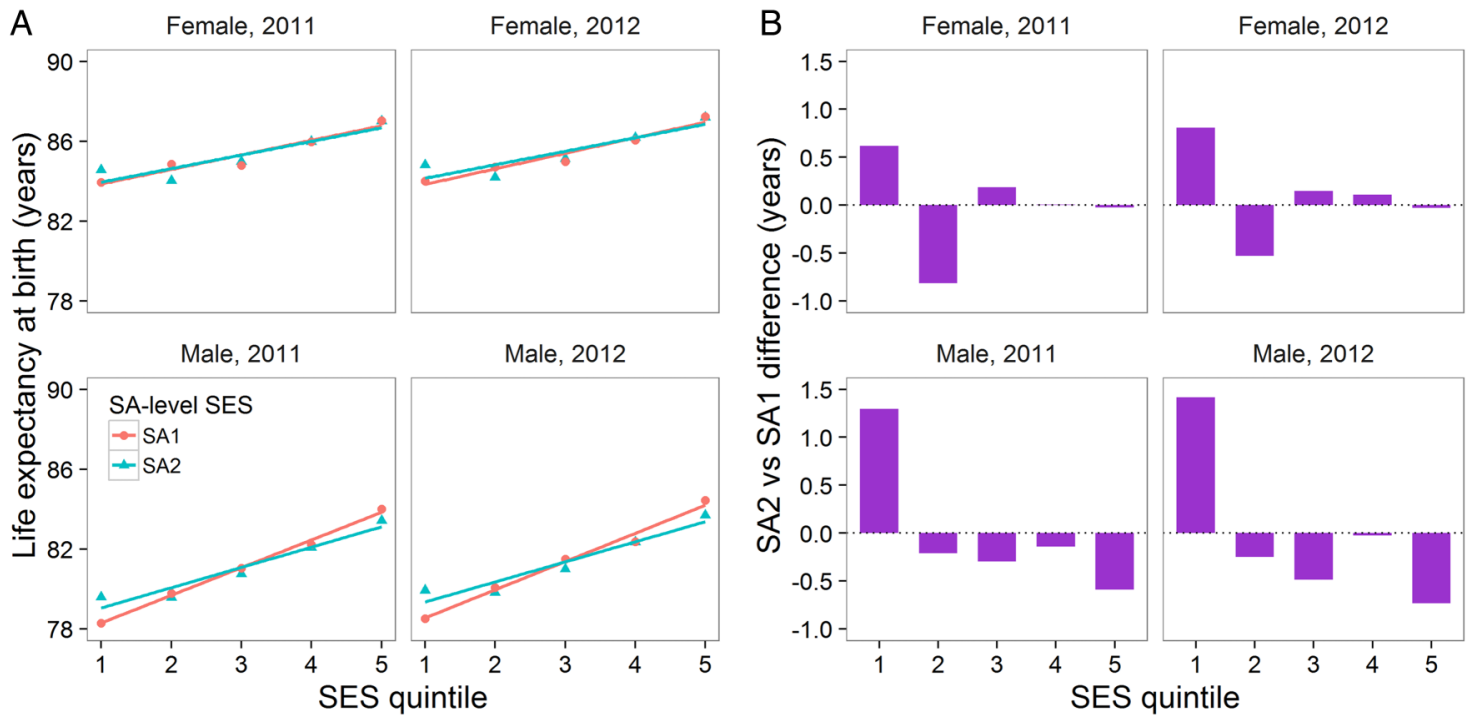

Figure 5 Comparison of life expectancy estimates calculated using SA1-level and SA2-level SES data for the Sydney metropolitan area. (A) Life expectancy at birth for males and females by SES quintile and year calculated using SA1-level and SA2-level SES data. (B) Differences in life expectancy by SES quintile between estimates calculated using SA1-level and SA2-level SES data. Bars show the difference in estimates calculated using SA2-level SES data minus estimates calculated using SA1-level SES data. 
expectancy between 2001 and 2012. We showed that, overall, standardised mortality rates declined, life expectancy increased and the patterns of the leading causes of death shifted in NSW during 2001-2012. We also showed that life expectancy varied by socioeconomic disadvantage, with larger disparities observed in men. Temporal analysis revealed that differences in life expectancy by socioeconomic disadvantage were stable between 2001 and 2012. When assessed by gender, gaps in life expectancy were sizeable and related to SES, but narrowed between 2001 and 2012. In contrast, remoteness effects on life expectancy were mostly small, with the largest differences observed in SES quintile 1, in 2012. Collectively, these findings demonstrated that, overall, life expectancy increased during 2001-2012, but remained strongly associated with socioeconomic disadvantage and gender, but less so with remoteness.

Our analysis revealed substantial changes in mortality between 2001 and 2012. Traditional life expectancy at birth increased by 1.9 and 3.0 years for females and males, respectively, indicating that the gain in life expectancy for males outpaced that of females; a finding that is comparable to recent trends in many OECD nations. ${ }^{18} \mathrm{~A}$ changing pattern in the leading causes of death was also observed over the study period, with substantial declines seen for CHD and cerebrovascular disease, and large relative increases observed for diabetes and dementia/Alzheimer's disease. The rises in mortality attributable to diabetes and dementia/ Alzheimer's disease is consistent with their emergence as more prominent chronic conditions, owing to increased longevity, declining burden of communicable diseases and changing patterns of obesity and inactivity. ${ }^{19} 20$

Life expectancy was strongly associated with socioeconomic disadvantage. A gradient of increasing life expectancy with increasing SES was observed in males and females, with a steeper gradient for males than females. The difference in life expectancy between the most and least disadvantaged quintiles was 3.8 years for males and 2.4 years for females (in major cities). To put into context, these differences are larger than the increases in life expectancy observed over the 12-year study period by each sex, respectively. Smaller socioeconomic differences in life expectancy in females have been observed in other countries, including the USA, South Korea, and some European nations, ${ }^{21-23}$ and indicate that the health impact of socioeconomic factors may be stronger in males than females. When assessed over time, the magnitudes of the differences between IRSD quintiles changed very little over the 12-year study period, with the exception of the global net gain in life expectancy in major city IRSD quintile 1 and the net decrease in life expectancy in regional/remote areas IRSD quintile 1. Although the size of the socioeconomic inequality in life expectancy has remained largely stable, it is encouraging that they have not widened, as they have performed in the USA and some European counties. $^{22-24}$
Disparities in life expectancy by sex were also sizeable. Females showed higher life expectancy across all IRSD quintiles, and by as much as nearly 5 years in 2012 . Higher life expectancy in females is observed globally and is thought to be attributable to biological factors, as well as modifiable factors such as lower levels of risk taking, smoking and alcohol consumption. ${ }^{25-27}$ Interestingly, the magnitude of the difference in life expectancy between males and females was related to IRSD quintile; being largest in the most disadvantaged quintiles and smallest in the most advantaged quintile, and suggests that socioeconomic factors also contribute to the gender gap. Encouragingly, the differences in life expectancy between females and males decreased over the study period by $\sim 1$ year for all SES-remoteness groups. These observed decreases in gender gaps over time are consistent with trends seen in other OECD countries. ${ }^{28}$

Remoteness was only minimally associated with life expectancy. In 2001, life expectancy was largely similar in major cities compared to equivalent IRSD quintiles in regional/remote areas. Similar patterns were observed in 2012, with the exception of IRSD quintile 1, which now showed a clear remoteness effect, manifested through the smaller and larger gains in life expectancy in regional/remote areas and major cities, respectively, for males and females. The distribution of IRSD index values is left-skewed, which means IRSD quintile 1 contains a wide-range of values. Therefore, it is possible that some of the observed differences could be explained by changes in the distribution of IRSD levels/ranks within the quintile. For example, it is possible that SA2s within major cities could have become relatively less disadvantaged while remaining in the same quintile while SA2s in regional and remote areas have become more disadvantaged.

The study had several limitations. The use of areabased SES, rather than individual-level information, may have led to potential misclassification of SES. The validation substudy allowed us to explore misclassificationmediated bias, revealing substantial differences in life expectancy (>1 year in some cases) when using larger, SA2-level IRSD compared to SA1-level IRSD. Crucially, it is likely that this bias would have been even greater had the comparison been made using an individual-level assessment of SES. The substudy also showed that the misclassification caused an overall attenuation of observed differences in life expectancy across the spectrum of IRSD quintiles. The inability to disentangle the individual effects of the constituent elements of the composite IRSD represents another limitation. For example, it is not possible to determine whether education or employment has greater effect on life expectancy. Life expectancy is an abstract measure, and although it is a useful summary measure of the mortality experience of a population and often used to represent the expected number of years to be lived by a newborn, it is based on current mortality rates, which are likely to 
change in the future. Finally, information on factors (such as biological, clinical and behavioural) that potentially mediate decreased life expectancy with socioeconomic disadvantage was lacking, preventing exploration of the mechanisms and pathways that lead to lower life expectancy.

\section{Conclusion}

In conclusion, standardised mortality rates declined substantially across all sex-SES-remoteness strata in NSW between 2001 and 2012, resulting in gains in life expectancy for all strata groups. The gains in males outpaced those of females. However, differences in life expectancy between IRSD quintiles were largely stable over time, with the exception of the relatively larger gains observed in IRSD quintile 1 in major cities, and importantly, the relatively smaller gains seen in IRSD quintile 1 in regional and remote areas. Socioeconomic disparities in life expectancy were wider in males than in females. Estimates of life expectancy that use SA2-level socioeconomic information probably underestimate any socioeconomic gradient. The study provides current estimates of differences in life expectancy by important sociodemographic factors, placing tangible values on the differences in life expectancy associated with socioeconomic inequalities.

Acknowledgements The authors thank the NSW Centre for Health Record Linkage and the Centre for Epidemiology and Evidence, NSW Ministry of Health, for the provision of access to the population health data used in the study.

Contributors ASS conceived and designed the study, conducted the data and statistical analysis, contributed to the interpretation of the findings and drafted the manuscript. LG and ST contributed the interpretation of the findings and the drafting of the manuscript. RAB contributed to the study design, data analysis and interpretation of the findings, and drafted the manuscript.

Funding This research received no specific grant from any funding agency in the public, commercial or not-for-profit sectors.

Competing interests None declared.

Ethics approval Ethical approval for this project was obtained from the NSW Population and Health Services Research Ethics Committee.

Provenance and peer review Not commissioned; externally peer reviewed.

Data sharing statement No additional data are available.

Open Access This is an Open Access article distributed in accordance with the Creative Commons Attribution Non Commercial (CC BY-NC 4.0) license, which permits others to distribute, remix, adapt, build upon this work noncommercially, and license their derivative works on different terms, provided the original work is properly cited and the use is non-commercial. See: http:// creativecommons.org/licenses/by-nc/4.0/

\section{REFERENCES}

1. Australian Institute of Health and Welfare. Australia's health 2014. Cat. no. AUS 178. 2014

2. World Health Organization. The determinants of health. 2016. http:// www.who.int/hia/evidence/doh/en/ (accessed 28 Jan 2016).
3. Elo IT. Social class differentials in health and mortality: patterns and explanations in comparative perspective. Annu Rev Sociol 2009;35:553-72.

4. Marmot M, Allen J, Bell R, et al. WHO European review of social determinants of health and the health divide. Lancet 2012;380:1011-29.

5. World Health Organization. Global Health Observatory. 2016. http:// www.who.int/gho/en/ (accessed 9 Jun 2016).

6. OECD. In it together: why less inequality benefits all. 2015 .

7. Australian Institute of Health and Welfare. Mortality inequalities in Australia 2009-2011. Bulletin no. 124. Cat. no. AUS 184. 2014.

8. Silcocks PB, Jenner DA, Reza R. Life expectancy as a summary of mortality in a population: statistical considerations and suitability for use by health authorities. $J$ Epidemiol Community Health 2001;55:38-43.

9. World Health Organization. International Classification of Diseases (ICD). 2016. http://www.who.int/classifications/icd/en/ (accessed 28 Jan 2016).

10. Australian Bureau of Statistics. Australian Statistical Geography Standard (ASGS). Cat. No. 1270.0.55.001.2011.

11. Australian Bureau of Statistics. Socio-Economic Indexes for Areas (SEIFA). Cat. No. 2033.0.55.001. 2011.

12. Australian Population and Migration Research Centre. ARIA (Accessibility/Remoteness Index of Australia). 2014. http://www. adelaide.edu.au/apmrc/research/projects/category/about_aria.html (accessed 7 Apr 2014).

13. Australian Institute of Health and Welfare. Leading causes of death. 2014. http://www.aihw.gov.au/deaths/leading-causes-of-death/ (accessed 28 Jan 2016).

14. Chiang CL. The life table and its construction. In: Introduction to stochastic processes in Biostatistics. New York: John Wiley \& Sons, 1968:189-214.

15. Chiang CL. Lifetable and its application. New York: Robert E. Krieger, 1984.

16. Stephens AS, Purdie S, Yang B, et al. Life expectancy estimation in small administrative areas with non-uniform population sizes: application to Australian New South Wales local government areas. BMJ Open 2013;3:e003710.

17. Bland JM, Altman DG. Statistical methods for assessing agreement between two methods of clinical measurement. Lancet 1986; $1: 307-10$

18. OECD. Life expectancy by sex and education level. In: Health at a Glance 2013, OECD Indicators. OECD Publishing, 2013, 26-27.

19. van Dieren S, Beulens JW, van der Schouw YT, et al. The global burden of diabetes and its complications: an emerging pandemic. Eur J Cardiovasc Prev Rehabil 2010;17(Suppl 1):S3-8.

20. World Health Organization. Global Health and Aging. 2011. http:// www.who.int/ageing/publications/global_health/en/ (accessed 2 Mar 2016).

21. Khang YH, Yang S, Cho HJ, et al. Decomposition of socio-economic differences in life expectancy at birth by age and cause of death among 4 million South Korean public servants and their dependents. Int J Epidemiol 2010;39:1656-66.

22. Mackenbach P. Health Inequalities: Europe in Profile. 2006. http:// www.who.int/social determinants/resources/european inequalities. pdf (accessed 2 Mar 2016).

23. Singh GK, Siahpush M. Widening socioeconomic inequalities in US life expectancy, 1980-2000. Int J Epidemiol 2006;35:969-79.

24. Chetty R, Stepner M, Abraham S, et al. The association between income and life expectancy in the United States, 2001-2014. JAMA 2016;315:1750-66.

25. Austad SN. Why women live longer than men: sex differences in longevity. Gend Med 2006;3:79-92.

26. Gjonca A, Tomassini C, Toson B, et al. Sex differences in mortality, a comparison of the United Kingdom and other developed countries. Health Stat Q 2005;26:6-16.

27. McCartney G, Mahmood L, Leyland AH, et al. Contribution of smoking-related and alcohol-related deaths to the gender gap in mortality: evidence from 30 European countries. Tob Control 2011;20:166-8.

28. Liu Y, Arai A, Kanda K, et al. Gender gaps in life expectancy: generalized trends and negative associations with development indices in OECD countries. Eur J Public Health 2013;23:563-8. 